\title{
COEFFICIENT STABILITY OF OPERATOR-DIFFERENCE SCHEMES WITH TIME VARIABLE OPERATORS
}

\author{
I.N. PANAYOTOVA \\ University of Rousse \\ Student St. 8, 7017 Rousse, Bulgaria \\ E-mail: jpan@ami.ru.acad.bg
}

\begin{abstract}
The problem of the coefficient stability for operator- difference schemes with variable operator is investigated. A priori coordinated estimates in the $L_{2}$-norm are obtained for differential-operator equations and operator-difference schemes. Estimates in the energy space $H_{A}$ for coefficient stability and stability with respect to the right-hand side and the initial data are proved under more strong assumptions for operator's perturbation.
\end{abstract}

\section{INTRODUCTION}

For many differential problem the coefficients of the equation may not be strictly defined. For example, this often happens if we get them by using some computational algorithm or as a result of physical experiment. This fact emphasizes the importance of investigation of schemes with perturbed coefficients. The difference scheme is called "coefficiently stable" if small perturbation of scheme's coefficients implies small changes of the solution.

The necessity of the coefficient stability was remarked in the beginning of the 60 years, in the papers of Tikhonov and Samarskii [4]. A priori estimates for the solution of the stationary problem, demonstrating continuous dependence on the perturbations of the right-hand side and on the perturbations of the operator (strong stability) can be found in the books $[1,9]$. The problem of coefficient stability for stationary differential equation is considered in Senatorov's articles [5,6]. A priori estimates for evolutionary equations with constant operators, when the operator, the right-hand side and the initial data were perturbed, are investigated in [2]. At the differential level, we note the article [10].

In $[7,8]$ a new measure for a perturbation of the unlimited operator $A^{*}=$ 
$A>0$ is introduced. With this measure the coefficient stability is proved under substantially weaker conditions then in the fundamental work [2].

In the paper, by means of the technics, introduced in [2], the problem of the coefficient stability when the operators depend on the time, are established. Under natural assumptions on operator's perturbation [7,8], a priori estimates in the $L_{2}$-norm for differential problem are obtained. Correspondingly coordinated estimates are proved for operator-difference schemes, after discretization in time. Estimates of strong stability for the scheme with weights in the energy space $H_{A}$ are obtained, if more strong assumptions [2] for operator's perturbation are assumed.

It is interesting to note that the estimates of strong stability in the $L_{2}-$ norm are proved without a standard requirement of Lipschitz continuity of the time dependent operator $A(t)$.

\section{COEFFICIENT STABILITY OF DIFFERENTIAL -OPERATOR EQUATIONS WITH VARIABLE OPERATOR}

Let $\mathrm{H}$ be a real finite dimensional Euclidean space with an inner product $(\cdot, \cdot)$ and a norm $\|\cdot\|$. For further investigations we will specify that $A=A(t)$ is dependent on the time, self-adjoint and positively defined operator in $\mathrm{H}$ :

$$
A^{*}(t)=A(t) \geq \delta E, \quad \delta>0 .
$$

We denote by $H_{R}, R^{*}=R>0$ a Hilbert space with an inner product $(y, v)_{R}=$ $(R y, v)$ and a norm $\|y\|_{R}=\sqrt{(y, y)_{R}}$.

Consider the Cauchy problem:

$$
\frac{d u}{d t}+A(t) u=f(t), \quad 0<t<T ; \quad u(0)=u_{0},
$$

where $f(t), u_{0}$ are given vectors. By $\widetilde{u}(t)$ we denote the solution of the problem with perturbed right-hand side, initial data and operator:

$$
\frac{d \widetilde{u}}{d t}+\widetilde{A}(t) \widetilde{u}=\widetilde{f}(t), \quad 0<t<T ; \quad \widetilde{u}(0)=\widetilde{u}_{0} .
$$

Our goal is to estimate the perturbation of the solution $z(t)=\widetilde{u}(t)-u(t)$ by means of perturbations of $f(t), u_{0}$ and operator $A(t)$. We will suppose about the perturbed operator $\widetilde{A}(t)$ the same properties as for the non-perturbed operator $A(t)$ :

$$
\widetilde{A}^{*}(t)=\widetilde{A}(t) \geq \widetilde{\delta} E, \quad \widetilde{\delta}>0 .
$$

The non-negative constant $\alpha[7,8]$ in the inequality

$$
|((\widetilde{A}(t)-A(t)) y, v)| \leq \alpha\|y\|_{\widetilde{A}(t)}\|v\|_{A(t)}, \quad \alpha \geq 0,
$$


will serve as a measure for operator's perturbation.

THEOREM 1. If conditions (1), (4) and (5) are fulfilled, then the following estimate for the perturbation of the solution $z(t)=\widetilde{u}(t)-u(t)$ holds:

$$
\begin{gathered}
\|\widetilde{u}(t)-u(t)\|^{2} \leq\left\|\widetilde{u}_{0}-u_{0}\right\|^{2}+\int_{0}^{t}\|\widetilde{f}(\theta)-f(\theta)\|_{A^{-1}(\theta)}^{2} d \theta \\
+\alpha^{2}\left(\left\|\widetilde{u}_{0}\right\|^{2}+\int_{0}^{t}\|\widetilde{f}(\theta)\|_{\widetilde{A}^{-1}(\theta)}^{2} d \theta\right) .
\end{gathered}
$$

Proof. Subtracting (2) from (3) we get the problem for $z(t)$ :

$$
\frac{d z}{d t}+A(t) z=(\widetilde{f}(t)-f(t))-(\widetilde{A}(t)-A(t)) \widetilde{u}, \quad 0<t<T ; \quad z(0)=\widetilde{u}_{0}-u_{0}
$$

By means of technics given in [2] we multiply equation (7) by $2 z$ in scalar way in $\mathrm{H}$ :

$$
\frac{d}{d t}\|z\|^{2}+2\|z\|_{A(t)}^{2}=2(\widetilde{f}(t)-f(t), z)-2((\widetilde{A}(t)-A(t)) \widetilde{u}, z) .
$$

Using the Cauchy-Schwarz inequality and condition (5) we obtain following estimates:

$$
\begin{gathered}
2(\widetilde{f}(t)-f(t), z) \leq\|\widetilde{f}(t)-f(t)\|_{A^{-1}(t)}^{2}+\|z\|_{A(t)}^{2}, \\
2((\widetilde{A}(t)-A(t)) \widetilde{u}, z) \leq 2 \alpha\|\widetilde{u}\|_{\widetilde{A}(t)}\|z\|_{A(t)} \leq \alpha^{2}\|\widetilde{u}\|_{\widetilde{A}(t)}^{2}+\|z\|_{A(t)}^{2} .
\end{gathered}
$$

Substituting inequalities obtained above in (8) we get:

$$
\frac{d}{d t}\|z\|^{2} \leq\|\widetilde{f}(t)-f(t)\|_{A^{-1}(t)}^{2}+\alpha^{2}\|\widetilde{u}\|_{\widetilde{A}(t)}^{2} .
$$

Integrating the last inequality with respect to time from 0 to $t$ we come to the following estimate:

$$
\|z(t)\|^{2} \leq\|z(0)\|^{2}+\int_{0}^{t}\|\widetilde{f}(\theta)-f(\theta)\|_{\widetilde{A}^{-1}(\theta)}^{2} d \theta+\alpha^{2} \int_{0}^{t}\|\widetilde{u}(\theta)\|_{\widetilde{A}(\theta)}^{2} d \theta .
$$

In order to estimate the last term in (9) we consider the perturbed equation (3). Multiplying (3) by $2 \widetilde{u}$ in scalar way we get the following results:

$$
\frac{d}{d t}\|\widetilde{u}\|^{2}+2\|\widetilde{u}\|_{\widetilde{A}(t)}^{2}=2(\widetilde{f}(t), \widetilde{u}) \leq\|\widetilde{u}\|_{\widetilde{A}(t)}^{2}+\|\widetilde{f}(t)\|_{\widetilde{A}^{-1}(t)}^{2} .
$$


Integrating this inequality with respect to time we come to the estimate:

$$
\int_{0}^{t}\|\widetilde{u}(\theta)\|_{\widetilde{A}(t)}^{2} d \theta \leq\|\widetilde{u}(0)\|^{2}+\int_{0}^{t}\|\widetilde{f}(\theta)\|_{\widetilde{A}^{-1}(\theta)}^{2} d \theta .
$$

Substituting (10) in (9) we finally come to the required a priori estimate (6). Theorem is proved.

\section{COEFFICIENT STABILITY OF TWO-LAYERED OPERATOR-DIFFERENCE SCHEMES WITH WEIGHTS AND VARIABLE OPERATOR}

In this part we will investigate the difference schemes obtained by discretization of the time in differential equation (2). Correspondingly coordinated estimates will be proved.

Let $\tau>0$ be a time step and $y_{n}=y\left(t_{n}\right), A_{n}=A\left(t_{n}\right), f_{n}=f\left(t_{n}\right), t_{n}=n \tau$. We will use the following notations:

$$
\begin{gathered}
y=y_{n}, \quad \hat{y}=y_{n+1}, \quad \check{y}=y_{n-1}, \\
y_{t}=(\hat{y}-y) / \tau, y_{\bar{t}}=(y-\check{y}) / \tau, y^{(\sigma)}=\sigma \hat{y}+(1-\sigma) y .
\end{gathered}
$$

For Cauchy problem (2) we set the difference scheme with weights:

$$
y_{t, n}+A_{n} y_{n}^{(\sigma)}=f_{n}, \quad n=0,1, \ldots ; \quad y_{0}=u_{0} .
$$

The difference scheme for the perturbed problem (3) is given as

$$
\widetilde{y}_{t, n}+\widetilde{A}_{n} \widetilde{y}_{n}^{(\sigma)}=\widetilde{f}_{n}, \quad n=0,1, \ldots ; \quad \widetilde{y}_{0}=\widetilde{u}_{0} .
$$

THEOREM 2. If conditions (1), (4) and (5) are fulfilled and $\sigma \geq 0.5$, then the solutions of schemes (11) and (12) satisfy the following estimate

$$
\begin{aligned}
\left\|\widetilde{y}_{n+1}-y_{n}\right\|^{2} \leq & \left\|\widetilde{u}_{0}-u_{0}\right\|^{2}+\sum_{k=0}^{n} \tau\left\|\widetilde{f}_{k}-f_{k}\right\|_{A_{k}^{-1}}^{2} \\
& +\alpha^{2}\left(\left\|\widetilde{u}_{0}\right\|^{2}+\sum_{k=0}^{n} \tau\left\|\widetilde{f}_{k}\right\|_{\widetilde{A}_{k}^{-1}}^{2}\right) .
\end{aligned}
$$

Proof. Subtracting (11) from (12) we get the problem for the perturbation of the solution $z_{n}=\widetilde{y}_{n}-y_{n}$ :

$$
z_{t, n}+A_{n} z_{n}^{(\sigma)}=\left(\widetilde{f}_{n}-f_{n}\right)-\left(\widetilde{A}_{n}-A_{n}\right) \widetilde{y}_{n}^{(\sigma)}, n=0,1, \ldots ; \quad z_{0}=\widetilde{u}_{0}-u_{0} .
$$


Multiplying equation (14) by $2 \tau z_{n}^{(\sigma)}$ in scalar way in $\mathrm{H}$ and using $\varepsilon$-inequality with condition (5) we obtain

$$
2 \tau^{2}(\sigma-0,5)\left\|z_{t, k}\right\|^{2}+\left\|z_{k+1}\right\|^{2} \leq\left\|z_{k}\right\|^{2}+\tau\left\|\widetilde{f}_{k}-f_{k}\right\|_{A_{k}^{-1}}^{2}+\alpha^{2} \tau\left\|\widetilde{y}_{k}^{(\sigma)}\right\|_{\widetilde{A}_{k}}^{2}
$$

From this, using condition $\sigma \geq 0,5$ and summing over all $k=0,1, \ldots n$, yields

$$
\left\|z_{n+1}\right\|^{2} \leq\left\|z_{0}\right\|^{2}+\sum_{k=0}^{n} \tau\left\|\widetilde{f}_{k}-f_{k}\right\|_{A_{k}^{-1}}^{2}+\alpha^{2} \sum_{k=0}^{n} \tau\left\|\widetilde{y}_{k}^{(\sigma)}\right\|_{\widetilde{A}_{k}}^{2} .
$$

In order to estimate the last term in the above inequality we over consider the perturbed scheme (12). Multiplying (12) by $2 \tau \widetilde{y}_{k}^{(\sigma)}$ in scalar way in $\mathrm{H}$ we get:

$$
2 \tau(\sigma-0,5)\left\|\widetilde{y}_{t}\right\|^{2}+\left\|\widetilde{y}_{k+1}\right\|^{2}+\tau\left\|\widetilde{y}_{k}^{(\sigma)}\right\|_{\widetilde{A}_{k}}^{2} \leq\left\|\widetilde{y}_{k}\right\|^{2}+\tau\left\|\widetilde{f}_{k}\right\|_{\widetilde{A}_{k}^{-1}}^{2}
$$

Then summing over all $k=0,1, \ldots, n$ we finally get the estimate:

$$
\sum_{k=0}^{n} \tau\left\|\widetilde{y}_{k}^{(\sigma)}\right\|_{\widetilde{A}_{k}}^{2} \leq\left\|\widetilde{u}_{0}\right\|^{2}+\sum_{k=0}^{n} \tau\left\|\widetilde{f}_{k}\right\|_{\widetilde{A}_{k}^{-1}}^{2}
$$

Substituting (16) in (15) we prove the validity of (13).

\section{COEFFICIENT STABILITY IN MORE STRONG ENERGY NORMS}

In order to obtain results in more strong energy norms, we will use a different measure of the perturbation. The non-negative constant $\alpha$ in the inequality

$$
\|(\widetilde{A}(t)-A(t) v)\| \leq \alpha\|\widetilde{A}(t) v\|, \quad \alpha \geq 0
$$

will be a measure of the perturbation of the operator $A(t)$. For further investigations we will assume that the operators $A(t)$ and $\widetilde{A}(t)$ are Lipschitz continuous with respect to time

$$
\begin{gathered}
|((A(t)-A(t-\tau)) v, v)| \leq \tau c_{0}(A(t-\tau) v, v), c_{0}=\text { const }>0, v \in H \\
|((\widetilde{A}(t)-\widetilde{A}(t-\tau)) v, v)| \leq \tau \widetilde{c}_{0}(\widetilde{A}(t-\tau) v, v), \widetilde{c}_{0}=\text { const }>0
\end{gathered}
$$

Firstly we will formulate the following auxiliary results. 
Lemma 3. [3] Suppose that operator $\widetilde{A}(t)$ is variable self-adjoint, positively defined operator and if satisfies condition (19) of Lipschitz continuity with respect to the time. Then provided $\sigma \geq 0,5$ the following a priori estimate holds for the solution of the perturbed scheme (12)

$$
\begin{aligned}
\left\|\widetilde{y}_{n+1}\right\|_{\widetilde{A}_{n}} \leq & M\left(\left\|\widetilde{u}_{0}\right\|_{\widetilde{A}_{0}}+\max _{0 \leq k \leq n}\left(\left\|\widetilde{f}_{k}\right\|_{\widetilde{A}_{k}^{-1}}+\left\|\widetilde{f}_{\bar{t}, k}\right\|_{\widetilde{A}_{k}^{-1}}\right)\right), \\
& M=\text { const }>0, f_{-1}=f_{0} .
\end{aligned}
$$

Lemma 4. Under the conditions of Lemma 3, we have a priori estimate in integral with respect to time norms for the solution of the perturbed scheme (12):

$$
\begin{aligned}
& \sum_{k=0}^{n} \tau\left\|\widetilde{A}_{k} \widetilde{y}_{k}^{(\sigma)}\right\|^{2} \leq\left\|\widetilde{u}_{0}\right\|_{\widetilde{A}_{0}}^{2}+\sum_{k=0}^{n} \tau\left\|\widetilde{f}_{k}\right\|^{2}+\widetilde{M}\left(\left\|\widetilde{u}_{0}\right\|_{\widetilde{A}_{0}}^{2}\right. \\
& \left.\quad+\max _{0 \leq k \leq n}\left(\left\|\widetilde{f}_{k}\right\|_{\widetilde{A}_{k}^{-1}}^{2}+\left\|\widetilde{f}_{\bar{t}, k}\right\|_{\widetilde{A}_{k}^{-1}}^{2}\right)\right), \quad \widetilde{M}=4 t_{n} M \widetilde{c}_{0} .
\end{aligned}
$$

Proof. Multiplying the perturbed scheme (12) by $2 \tau \widetilde{A}_{n} \widetilde{y}_{n}^{(\sigma)}$ in scalar way in $H$ and applying equality $\widetilde{y}_{n}^{(\sigma)}=\tau(\sigma-0,5) \widetilde{y}_{t}+\widetilde{y}^{(0,5)}$, we have the energy identity:

$$
\begin{gathered}
2 \tau^{2}(\sigma-0,5)\left(\widetilde{A}_{n} \widetilde{y}_{t}, \widetilde{y}_{t}\right)+\left(\widetilde{A}_{n} \widetilde{y}_{n+1}, \widetilde{y}_{n+1}\right)+2 \tau\left\|\widetilde{A}_{n} \widetilde{y}_{n}^{(\sigma)}\right\|^{2} \\
=\left(\widetilde{A}_{n} \widetilde{y}_{n}, \widetilde{y}_{n}\right)+2 \tau\left(\widetilde{A}_{n} \widetilde{y}_{n}^{(\sigma)}, \widetilde{f}_{n}\right) .
\end{gathered}
$$

Using that $\sigma \geq 0,5$, the Cauchy-Schwarz inequality and Lipschitz continuity for the operator $\widetilde{A}(t)$ we get the recurrent formula:

$$
\tau\left\|\widetilde{A}_{k} \widetilde{y}_{k}^{(\sigma)}\right\|^{2}+\left\|\widetilde{y}_{k+1}\right\|_{\widetilde{A}_{k}}^{2} \leq\left(1+\tau \widetilde{c}_{0}\right)\left\|\widetilde{y}_{k}\right\|_{\widetilde{A}_{k-1}}^{2}+\tau\left\|\widetilde{f}_{k}\right\|^{2} .
$$

Summing over all $k=1, \ldots, n$ we obtain the following inequality:

$$
\sum_{k=1}^{n} \tau\left\|\widetilde{A}_{k} \widetilde{y}_{k}^{(\sigma)}\right\|^{2} \leq\left\|\widetilde{y}_{1}\right\|_{\widetilde{A}_{0}}^{2}+\widetilde{c}_{0} \sum_{k=1}^{n} \tau\left\|\widetilde{y}_{k}\right\|_{\widetilde{A}_{k-1}}^{2}+\sum_{k=1}^{n} \tau\left\|\widetilde{f}_{k}\right\|^{2} .
$$

From the energy identity (22) when $n=0$ we have the inequality:

$$
\tau\left\|\widetilde{A}_{0} \widetilde{y}_{0}^{(\sigma)}\right\|^{2}+\left\|\widetilde{y}_{1}\right\|_{\widetilde{A}_{0}}^{2} \leq\left\|\widetilde{y}_{0}\right\|_{\widetilde{A}_{0}}^{2}+\tau\left\|\widetilde{f}_{0}\right\|^{2} .
$$

Substituting (24) in (23) we come to the estimate:

$$
\sum_{k=0}^{n} \tau\left\|\widetilde{A}_{k} \widetilde{y}_{k}^{(\sigma)}\right\|^{2} \leq\left\|\widetilde{y}_{0}\right\|_{\widetilde{A}_{0}}^{2}+\sum_{k=0}^{n} \tau\left\|\widetilde{f}_{k}\right\|^{2}+\widetilde{c}_{0} \sum_{k=1}^{n} \tau\left\|\widetilde{y}_{k}\right\|_{\widetilde{A}_{k-1}}^{2} .
$$


In order, to estimate the last term in above inequality we apply Lemma 3 and since $\widetilde{M}=4 t_{n} M \widetilde{c}_{0}$ is a positive constant we finally get the required estimate (21). Lemma is proved.

THEOREM 5. Suppose the operators $A(t)$ and $\widetilde{A}(t)$ are variable, self-adjoint, positively defined operators and they satisfy conditions (17), (18) and (19). Then,when $\sigma \geq 0.5$, the solutions of schemes (11) and (12) satisfy the estimates

$$
\begin{gathered}
\left\|\widetilde{y}_{n+1} \quad-y_{n+1}\right\|_{A_{n}}^{2} \leq M_{1}^{2}\left(\left\|\widetilde{u}_{0}-u_{0}\right\|_{A_{0}}^{2}+\sum_{k=0}^{n} \tau\left\|\widetilde{f}_{k}-f_{k}\right\|^{2}\right. \\
\left.+\alpha^{2} \sum_{k=0}^{n} \tau\left\|\widetilde{A}_{k} \widetilde{y}_{k}^{(\sigma)}\right\|^{2}\right), \quad M_{1}=\rho^{N_{0}}=e^{0,5 c_{0} T} \\
\sum_{k=0}^{n} \tau\left\|\widetilde{A}_{k} \widetilde{y}_{k}^{(\sigma)}\right\|^{2} \leq\left\|\widetilde{u}_{0}\right\|_{\widetilde{A}_{0}}^{2}+\sum_{k=0}^{n} \tau\left\|\widetilde{f}_{k}\right\|^{2} \\
+\widetilde{M}\left(\left\|\widetilde{u}_{0}\right\|_{\widetilde{A}_{0}}^{2}+\max _{0 \leq k \leq n}\left(\left\|\widetilde{f}_{k}\right\|_{\widetilde{A}_{k}^{-1}}^{2}+\left\|\widetilde{f}_{\bar{t}, k}\right\|_{\widetilde{A}_{k}^{-1}}^{2}\right)\right) .
\end{gathered}
$$

Proof. Multiplying the equation (14) by $2 \tau z_{t}$ in scalar way in $H$ we get the recurrent formula:

$$
\begin{aligned}
\left\|z_{k+1}\right\|_{A_{k}}^{2} & \leq \rho^{2}\left\|z_{k}\right\|_{A_{k-1}}^{2}+\tau\left\|\widetilde{f}_{k}-f_{k}\right\|^{2}+\tau\left\|\left(\widetilde{A}_{k}-A_{k}\right) \widetilde{y}_{k}^{(\sigma)}\right\|^{2} \\
\rho & =e^{0,5 \tau c_{0}}, z_{k}=\widetilde{y}_{k}-y_{k} .
\end{aligned}
$$

Specially for $n=0$ we have:

$$
\left\|z_{1}\right\|_{A_{0}}^{2} \leq\left\|z_{0}\right\|_{A_{0}}^{2}+\tau\left\|\widetilde{f}_{0}-f_{0}\right\|^{2}+\tau\left\|\left(\widetilde{A}_{0}-A_{0}\right) \widetilde{y}_{0}^{(\sigma)}\right\|^{2} .
$$

Summing over all $k=0,1, \ldots, n$ we get the estimate:

$$
\left\|z_{n+1}\right\|_{A_{n}}^{2} \leq \rho^{2 n}\left(\left\|z_{0}\right\|_{A_{0}}^{2}+\sum_{k=0}^{n} \tau\left\|\widetilde{f}_{k}-f_{k}\right\|^{2}+\sum_{k=0}^{n} \tau\left\|\left(\widetilde{A}_{k}-A_{k}\right) \widetilde{y}_{k}^{(\sigma)}\right\|^{2}\right) .
$$

Finally if we use the estimate (17) for the perturbation of the operator $A(t)$ and Lemma 4 we complete the proof. 
Author expresses many thanks to prof. Matus for helpful discussions and his constant attention to this work.

\section{REFERENCES}

[1] A.A. Samarskii, Theory of difference schemes, Nauka, Moscow, 1977, (in Russian).

[2] A.A. Samarskii, P.N. Vabishchevich, P.P. Matus, Strong stability of differential-operator and operator-difference schemes, Dokl.RAS., 356, No 4 (1997), P. 455-457.

[3] A.A. Samarskii, P.N. Vabishchevich, P.P. Matus, Difference schemes with operator multipliers, Minsk, 1998

[4] A.N. Tikhonov, A.A. Samarskii, On uniform difference schemes, J.Vychisl.Mathem.i Mathem. Fiz., 1, (1961), P. 5-64.

[5] P.K. Senatorov, Coefficient stability of the solution of the ordinary differential equations of second level, Differential equations, 7, No 4 (1971), P. 754-758.

[6] P.K. Senatorov, On coefficient stability of the solution of Dirichlet problem for the equation $\operatorname{div}\{k(x)$ gradu $\}=-f(x)$, Differential equations, 7, No 10 (1971), P. 18691875.

[7] P.P. Matus, I.N. Panayotova, On coefficient stability of difference schemes, Proceedings of CFDM'98, Minsk, Belarus, P. 121-129.

[8] P.P. Matus, I.N. Panayotova, Strong stability of differential-operator equations and operator-difference schemes, Differential equations, 35, No 2 (1999) (will appear).

[9] S.G. Mikhlin, Some aspects of the theory of mistakes, Leningrad State University, Leningrad, 1988 (in Russian).

[10] U. Stryte, Difference schemes for parabolic equations on general type with weak solutions, Differential equations, 22, No 7 (1986), P. 1246-1260. 